\title{
Detector-Agnostic Phase-Space Distributions
}

\author{
J. Sperling $\odot,{ }^{1, *}$ D. S. Phillips, ${ }^{2}$ J. F. F Bulmer, ${ }^{2}$ G. S. Thekkadath, ${ }^{2}$ A. Eckstein, ${ }^{2}$ T. A. W. Wolterink, ${ }^{2}$ J. Lugani, ${ }^{2}$ \\ S. W. Nam, ${ }^{3}$ A. Lita, ${ }^{3}$ T. Gerrits, ${ }^{3}$ W. Vogel, ${ }^{4}$ G. S. Agarwal, ${ }^{5}$ C. Silberhorn, ${ }^{1}$ and I. A. Walmsley ${ }^{2}$ \\ ${ }^{1}$ Integrated Quantum Optics Group, Applied Physics, University of Paderborn, 33098 Paderborn, Germany \\ ${ }^{2}$ Clarendon Laboratory, University of Oxford, Parks Road, Oxford OX1 3PU, United Kingdom \\ ${ }^{3}$ National Institute of Standards and Technology, 325 Broadway, Boulder, Colorado 80305, USA \\ ${ }^{4}$ Institut für Physik, Universität Rostock, Albert-Einstein-Straße 23, D-18059 Rostock, Germany \\ ${ }^{5}$ Texas A\&M University, College Station, Texas 77845, USA
}

(Received 28 April 2019; published 9 January 2020)

\begin{abstract}
The representation of quantum states via phase-space functions constitutes an intuitive technique to characterize light. However, the reconstruction of such distributions is challenging as it demands specific types of detectors and detailed models thereof to account for their particular properties and imperfections. To overcome these obstacles, we derive and implement a measurement scheme that enables a reconstruction of phase-space distributions for arbitrary states whose functionality does not depend on the knowledge of the detectors, thus defining the notion of detector-agnostic phase-space distributions. Our theory presents a generalization of well-known phase-space quasiprobability distributions, such as the Wigner function. We implement our measurement protocol, using state-of-the-art transition-edge sensors without performing a detector characterization. Based on our approach, we reveal the characteristic features of heralded single- and two-photon states in phase space and certify their nonclassicality with high statistical significance.
\end{abstract}

DOI: 10.1103/PhysRevLett.124.013605

Introduction.-The characterization of quantum light is a main challenge one encounters when implementing classically infeasible tasks, such as quantum communication protocols [1-3]. On a more fundamental level, studying the peculiarities of quantized radiation fields leads to a profound understanding of the role of quantum physics in nature in general, and how it is distinct from classical wave theories in particular. As in classical systems, quantumoptical phase-space distributions offer a versatile instrument to directly visualize unique features of nonclassical light, such as demonstrated for squeezing [4-6]. Moreover, negativities in certain phase-space functions directly point at quantum properties of light; see, e.g., Refs. [7-12]. For the above reasons, the representation of quantum light in phase space is one of the most frequently applied methods to characterize nonclassical light.

However, the estimation of phase-space distributions from experimental data is a cumbersome task. Consequently, this reconstruction problem inspired a wide range of research [13-15], leading to sophisticated analytical tools, such as solving inversion problems [16,17], employing diverging pattern functions $[18,19]$, performing maximum-likelihood estimations [20-22], and using data pattern recognition $[23,24]$. In addition, each family of detection devices has to be equipped with its own precise model to reliably extract information about phase-space functions [13-15]. This treatment comprises a comprehensive analysis that assesses (i) how a detector responds to incident light [25,26], including, e.g., nonlinear detection responses [27,28], and (ii) how the light absorption is influenced by a number of possible imperfections, e.g., efficiencies $[29,30]$. Moreover, applying these methods can also require universally applicable, yet rather demanding theoretical and experimental techniques in practice, such as performing detector tomography and calibration [31-39].

Despite these challenges, phase-space distributions constitute a highly successful approach to revealing nonclassical properties of light [7-12]. For example, s-parametrized quasiprobabilities [40,41], as well as their non-Gaussian generalizations $[42,43]$, can exhibit negativities that are incompatible with classical light. Even if a phase-space function does not exhibit negativities, observable patterns render it possible to identify quantum features, for instance, via the non-negative Husimi function [44-46] or through marginal distributions $[47,48]$. Because of its success, the concept of phase-space functions has been further extended to other physical scenarios; see Refs. [49,50]. To name a few, atomic ensembles [51-54] and entanglement [55-57] have been successfully characterized using quasiprobability distributions. Nevertheless, there remains a dependency on well-defined detection schemes and reconstruction algorithms.

In this contribution, we circumvent the reconstruction problem by devising a measurement protocol that results in detector-agnostic phase-space (DAPS) distributions, which can be directly estimated, encompass known 


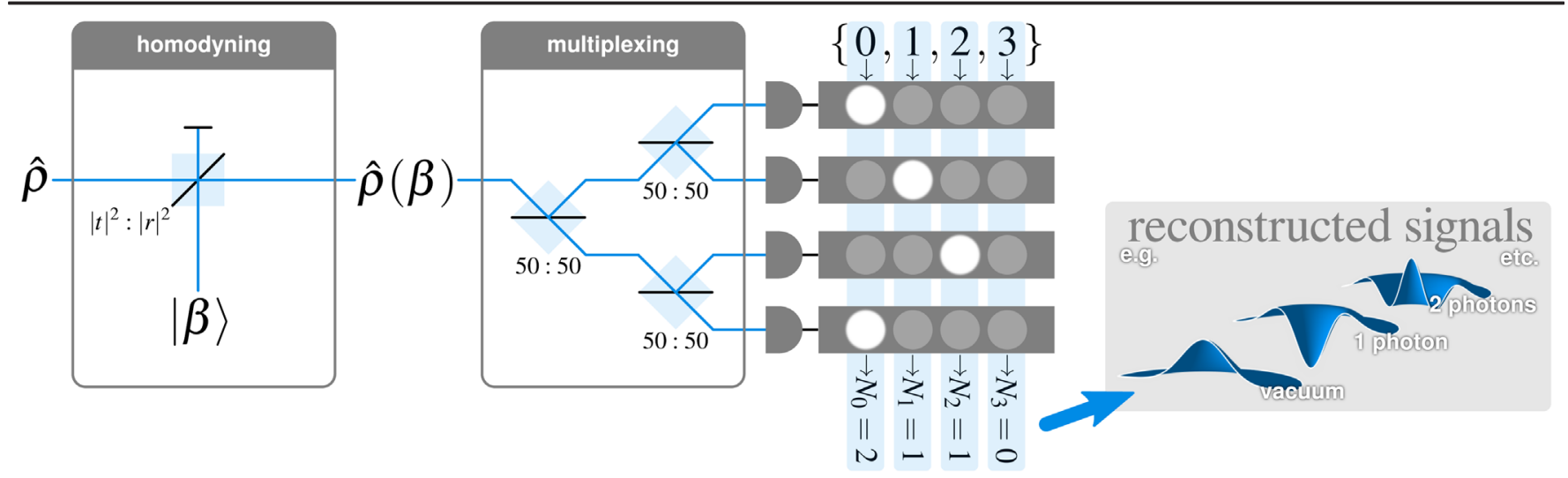

FIG. 1. Protocol overview. A signal state $\hat{\rho}$ is mixed on a $|t|^{2}:|r|^{2}$ beam splitter with a LO $|\beta\rangle$ in an unbalanced homodyning configuration. The resulting state $\hat{\rho}(\beta)$ is fed into a multiplexing scheme (shown for $S=2$ steps). Each output beam is measured with a detector that can produce some outcomes (here, $\mathcal{K}=\{0, \ldots, K=3\}$ ). The resulting statistics $c_{N_{0}, \ldots, N_{K}}(\beta)$ is obtained, where $N_{k}$ counts the number of outcomes $k$ and $N_{0}+\cdots+N_{K}=N$. From the measured data, we directly estimate our generalized phase-space distributions, cf., the first line in Eq. (2).

quasiprobabilities, and apply to arbitrary quantum states of light. We demonstrate our scheme with transition-edge sensors (TESs), which have sophisticated physics underlying their operation, and analyze our data without relying on any specific detector models. Our DAPS functions reveal nonclassical features expected from our heralded multiphoton states with high statistical significance. Moreover, the measurement of vacuum alone enables us to predict the unique structures of DAPS distributions as demonstrated for our experimentally generated states.

Theory framework.-Our measurement scheme is a combination of unbalanced homodyning [58] and a multiplexed detection layout [59]; see Fig. 1. A signal light field, $\hat{\rho}$, is mixed with a local oscillator (LO), $|\beta\rangle$, on a beam splitter. One of the output states, represented through $\hat{\rho}(\beta)$, is injected into a multiplexing scheme that consists of $S$ steps. In each step, light is split into output fields with the same intensity, which then can be split again. The finally obtained $N=2^{S}$ output beams are individually measured with unknown detectors, which are not specified but assumed to operate in the same manner. Each detector returns one of the possible outcomes $\mathcal{K}=\{0, \ldots, K\}$. In Refs. [60,61], we have shown for the multiplexing part that independently of the detector response, the probability to simultaneously measure $N_{k}$ times the outcome $k$ ( $\forall k \in \mathcal{K}$ ) follows a quantum version of the multinomial distribution; its generalization to $\hat{\rho}(\beta)$ reads

$$
c_{N_{0}, \ldots, N_{K}}(\beta)=\left\langle: \frac{N !}{N_{0} ! \cdots N_{K} !} \hat{\pi}_{0}^{N_{0}} \cdots \hat{\pi}_{K}^{N_{K}}:\right\rangle_{\hat{\rho}(\beta)},
$$

where $: \cdots:$ denotes the normal ordering and $\{\hat{\pi}\}_{k \in \mathcal{K}}$ is the unknown positive operator-valued measure of the detectors.

The only assumptions made are a balanced splitting in the multiplexing and identical response functions for the $N$ detectors, including all imperfections. We can account for deviations from both assumptions by including a systematic error, directly estimated from asymmetries in the measured data; see Supplemental Material (SM) for details [62].

A probability distribution is entirely characterized through its generation function, which can be expressed as

$$
\begin{aligned}
g_{z_{0}, \ldots, z_{K}}(\beta) & =\sum_{N_{0}, \ldots, N_{K}} z_{0}^{N_{0}} \cdots z_{K}^{N_{K}} c_{N_{0}, \ldots, N_{K}}(\beta) \\
& =\left\langle:\left(z_{0} \hat{\pi}_{0}+\cdots+z_{K} \hat{\pi}_{K}\right)^{N}:\right\rangle_{\hat{\rho}(\beta)},
\end{aligned}
$$

for $z_{0}, \ldots, z_{K} \in \mathbb{R}$. The second line is a result of the multinomial form of the statistics in Eq. (1). One salient feature is that classical light fields have a non-negative generation function $g_{z_{0}, \ldots, z_{K}}$. To see this, first recall that a classical light field is described through a non-negative Glauber-Sudarshan distribution [66,67], which is not affected by displacements and describes a state as a statistical mixture of coherent states. Furthermore, for all even $N$, we can define the operator $\hat{f}=\hat{f}^{\dagger}=\left(z_{0} \hat{\pi}_{0}+\right.$ $\left.\cdots+z_{K} \hat{\pi}_{K}\right)^{N / 2}$. Since for any non-negative GlauberSudarshan function $\left\langle: \hat{f}^{\dagger} \hat{f}:\right\rangle \geq 0$ holds true [63,68-70], we conclude

$$
g_{z_{0}, \ldots, z_{K}}(\beta) \stackrel{\text { cl. }}{\geq 0} .
$$

A violation of this inequality certifies the nonclassicality of the signal light, $\hat{\rho}$. We can also define a special case of this generating function,

$$
G_{z}(\beta)=g_{1, z, z^{2}, \ldots, z^{K}}(\beta) .
$$

Similarly to the expression in Eq. (2), $G_{z}$ is straightforwardly estimated from the measured detector outcomes $c_{N_{0}, \ldots, N_{K}}(\beta)$ by setting $z_{k}=z^{k}$, and $G_{z}$ is non-negative for classical light. 
As an example, we may consider photocounting [25]. Although this model is not required for our approach and does not apply to our experiment (TESs have a finite photon-number resolution, a non-unit detection efficiency, and a nonlinear response function [61]), it demonstrates how $G_{z}$ generalizes the concept of well-known phase-space distributions. For photocounting, we find $[62,64]$

$$
G_{z}(\beta)=\left\langle: e^{-[1-z] \eta \hat{n}}:\right\rangle_{\hat{\rho}(\beta)}=\frac{\pi(1-s)}{2} P\left(\frac{r}{t} \beta ; s\right),
$$

with $\eta$ and $\hat{n}$ being the efficiency and the photon-number operator, respectively, and $s=1-2 /\left[\eta|t|^{2}(1-z)\right]$ [71]. Thus, $G_{z}(\beta)$ resembles the $s$-parametrized distributions $P(r \beta / t ; s)[40,41]$. Beyond photoelectric detectors, we refer to $g_{z_{0}, \ldots, z_{K}}$ and $G_{z}$ as DAPS distributions as Eqs. (2) and (4) apply without any knowledge of the measurement operators $\left\{\hat{\pi}_{k}\right\}_{k \in \mathcal{K}}$. In this context, it is worth emphasizing that the first line in Eq. (2) enables the estimation of our DAPS distributions as a result of the measured coincidence statistics $c_{N_{0}, \ldots, N_{K}}(\beta)$ alone.

Implementation.-By implementing a single multiplexing step, $N=2^{S}=2$ for $S=1$, we demonstrate how to apply our theoretical framework of DAPS distributions. To realize our protocol in Fig. 1, we produce heralded photon states $\hat{\rho}$ and different LO amplitudes $\beta$. The detectors used for the multiplexing measurement and the heralding are TESs, which count photons up to a maximal number $K$. In the following, we describe the experimental setup [62].

Femtosecond pulses with a $100 \mathrm{kHz}$ repetition rate from a titanium sapphire laser are coupled into two separate, periodically poled potassium titanyl phosphate (PPKTP) wave guides. Both pulses are filtered to a full-width at halfmaximum of $\pm 2 \mathrm{~nm}$ using angle-tuned bandpass filters. With the first PPKTP wave guide, prepare the signal $\hat{\rho}$. When filtering the pump at $775 \mathrm{~nm}$ the wave guide produces two-mode squeezed vacuum in approximately a single spatiotemporal mode via type-II parametric down conversion (PDC) [72]. The signal mode at $1554 \mathrm{~nm}$ and the herald mode at $1547 \mathrm{~nm}$ are separated with a polarizing beam splitter, then filtered and coupled into single-mode optical fibers. The herald mode is then sent to a single TES detector. With the second PPKTP wave guide, we prepare the LO. In contrast to the signal state generation, we filter the pump at $783 \mathrm{~nm}$ and stimulate the PDC process by seeding it with 2 ns pulses carved with an electro-optic modulator from a $1580 \mathrm{~nm}$ continuous-wave laser. Because of the strong seed signal, this nonlinear mixing generates coherent light to an excellent approximation in the polarization mode orthogonal to the seed [73]. We separate the LO from the seed with a polarizing beam splitter, then pass through a bandpass filter at $1554 \mathrm{~nm}$. By pumping the two wave guides at different wavelengths, we are able to create a LO that is well mode matched to the signal using a seed laser that is detuned from the heralding mode. This avoids a potential source of noise due to the seed laser passing through the filters for the heralding TES. The generated LO is attenuated to the single-photon level and coupled into single-mode optical fiber. Crucially, this process prepares a LO with Poissonian photon statistics with a measured second-order correlation function $g^{(2)}(0)$ of $1.005 \pm 0.002$.

Finally, the LO $|\beta\rangle$ and signal $\hat{\rho}$ are combined on a 90:10 fiber beam splitter. We consider the port that uses $|r|^{2}=$ $10 \%$ of the LO and transmits $|t|^{2}=90 \%$ of the signal. The light from this port, $\hat{\rho}(\beta)$, is then impinged on a 50:50 fiber beam splitter for realizing a multiplexing step; both outputs are then sent to two separate TESs. See Fig. 1.

Our experiment uses three TES detectors that can have efficiencies above $\eta=90 \%$ [74]. TESs are superconducting photon-number-resolving detectors that we operate in a dilution refrigerator at a temperature of around $80 \mathrm{mK}$. Their response is amplified using an array of superconducting quantum interference devices [75], followed by further amplification and filtering at room temperature. This electrical signal is read by an analogue-to-digital converter and processed using a matched filter technique [76], which outputs a single value when triggered by a clock signal from the laser. We bin these values to assign a photon number. It should be noted that it is possible to extract slightly more accurate estimates of photon number, however, using more sophisticated signal processing techniques, yet without affecting the applicability of the DAPS distribution approach $[61,77]$.

We record the binned outcome at all three TESs for various LO amplitudes $\left(|\beta|^{2}\right.$ from 0 to $\sim 28$ in steps of $\left.\sim 1\right)$. The amplitude is controlled by varying the seed laser power. To obtain data for a specific heralded state $\hat{\rho}$, we consider the subset of trials with the appropriate detection outcome (i.e., heralding bin $k_{h}$ ) at the herald TES.

Verification of nonclassicality.-In a first step, we apply our DAPS distribution to uncover nonclassical features of our prepared states through the violation of condition (3). The optimal negativity we obtain from the DAPS function [Eq. (2)] is given by the minimum

$$
g_{\min }=\min _{\beta} \min _{\substack{z_{0}, \ldots, z_{K}: \\\left|z_{0}\right|^{2}+\cdots+\left|z_{K}\right|^{\leq} \leq 1}} g_{z_{0}, \ldots, z_{K}}(\beta) .
$$

To assess the quality of this approach, we compared our verification of nonclassicality with other methods. In Ref. [60], we demonstrated that a correlation matrix, $M$, obtained from the measured statistics in Eq. (1), is positive semidefinite for classical light, described through a nonnegative minimal eigenvalue $\mu_{\min }$ of $M$. The resulting notion of submultinomial light, $\mu_{\min }<0$, was shown to be a better figure of merit than other means of verifying nonclassicality [61], such as sub-Poisson light [78,79] and sub-binomial light $[80,81]$.

The comparison of $g_{\min }$ and $\mu_{\min }$ for our data is shown in Table I for different heralding bins $k_{h}$. For the heralded 
TABLE I. For different heralding outcomes, $k_{h}$, we show that the nonclassicality criteria $\mu_{\min }<0$ and $g_{\min }<0 . g_{\min }$ is defined in Eq. (6). $\mu_{\min }$ is the minimal eigenvalue to the second-order correlation matrix $M$ defined in Eq. (6) of Ref. [60]; see also SM [62]. "-0" indicates a slightly negative mean value that rounds to 0.

\begin{tabular}{lcc}
\hline \hline$k_{h}$ & $\mu_{\min }$ & $g_{\min }$ \\
\hline 0 & $(-0 \pm 9) \times 10^{-4}$ & $(-0 \pm 2) \times 10^{-9}$ \\
1 & $-0.15 \pm 0.03$ & $-0.026 \pm 0.003$ \\
2 & $-0.10 \pm 0.03$ & $-0.017 \pm 0.003$ \\
\hline \hline
\end{tabular}

one-photon (two-photon) states, we confirm $g_{\min }<0$ with 9 (6) standard deviations, while the submultinomial behavior is less significant, 5 (3) standard deviations. For the vacuum state, i.e., $k_{h}=0$, both measures are consistent with the classical expectation, $g_{\min }=0=\mu_{\min }$.

Reconstructed distributions.-From the data, we can directly estimate our DAPS distributions. The results of our extended analysis are shown in Fig. 2. The estimation procedure is this: we run the experiment twice, once with the signal blocked and once with the signal unblocked.

To have a full detector-agnostic approach, we first define a detector-independent coherent amplitude,

$\left|\beta^{(\mathrm{DI})}\right|=\sqrt{\sum_{N_{0}, \ldots, N_{K}}\left[0 N_{0}+\cdots+K N_{K}\right] c_{N_{0}, \ldots, N_{K}}^{(\mathrm{vac})}(\beta)}$,

which is given by the statistics $c_{N_{0}, \ldots, N_{K}}^{(\mathrm{vac})}(\beta)$ measured by blocking the signal [62]. In case of photocounting, this gives $\left|\beta^{(\mathrm{DI})}\right|=\sqrt{\eta}|r||\beta|$. As we do not record a phase, we consider full phase randomization. This does not affect the DAPS distributions of our heralded photon states. In Fig. 2, our DAPS distributions $G_{z}$ are shown as a function of the amplitude in Eq. (7), determined by means of the vacuum measurement.

The same measurement renders it possible to theoretically predict the DAPS distribution of arbitrary states.
Namely, a general DAPS distribution can be described as a convolution of the measured vacuum distribution $G_{z}^{(\mathrm{vac})}$ and the Glauber-Sudarshan distribution $P\left(\beta^{\prime} ; 1\right)$ of the state under study [62],

$$
G_{z}(\beta)=\int d^{2} \beta^{\prime} P\left(\beta^{\prime} ; 1\right) G_{z}^{(\mathrm{vac})}\left(\beta-\frac{t}{r} \beta^{\prime}\right) .
$$

In our case, the Gaussian shape of $G_{z}^{(\mathrm{vac})}$ implies that heralded single-photon (two-photon) states should follow a Gaussian distribution multiplied with a first-order (secondorder) polynomial in $\left|\beta^{(\mathrm{DI})}\right|^{2}$. In Fig. 2, this prediction (dashed lines) is confirmed as it correctly represents the DAPS distributions of the measured heralded photon states. The heralding to $k_{h}=1$ gives a characteristic dip at the origin $\left|\beta^{(\mathrm{DI})}\right|=0$, and the two-photon case, $k_{h}=2$, leads to additional oscillations together with the appearance of a peak at the origin. We emphasize that the functional behavior $\beta \mapsto G_{z}(\beta)$ depends on the measurement operators, but the estimation of $G_{z}(\beta)$ is done without any specification of the detector operators, according to the first line in Eq. (2). Moreover, we are able to characterize defining features of other states without any other prior knowledge about the detectors from the data obtained using the vacuum state input [Eq. (8)].

Based on our reconstruction, we were able to determine a number of other properties of the experimentally produced states [62]. For instance, we can determine how well the DAPS distributions enable us to perform a quantum state discrimination task. The single- and two-photon states [plots (b) and (c) in Fig. 2] can be distinguished from each other with more than $98 \%$ certainty. Furthermore, we found that, for $z<-2.4$, the central dip of $G_{z}$ becomes negative, similar to the behavior of other phase-space quasiprobabilities. The negativity has the highest statistical significance for $z=-4.85$, where $G_{z}(0)=-0.51 \pm 0.08$ is more than 6 standard deviations below the classical threshold of 0 .
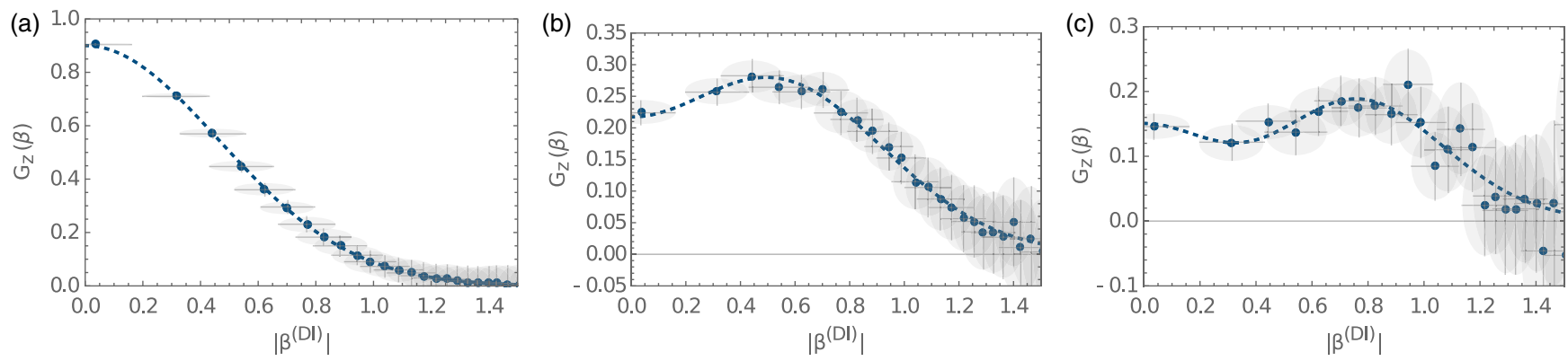

FIG. 2. Reconstructed DAPS distributions $G_{z}(\beta)$ [Eq. (4)] as a function of $\left|\beta^{(\mathrm{DI})}\right|$ [Eq. (7)]. We choose $z=-1.5$ as it would correspond to a Wigner function $(s=0)$ in the case of photocounting under the assumption of almost no loss, $\eta \approx 90 \%$. From left to right, (a)-(c), heralded $k_{h}=0,1,2$-photon states are shown. The dashed lines show the fit to a model inferred from the data obtained by blocking the signal [Eq. (8)]. The defining structures of the heralded (b) single-photon and (c) two-photon states are the oscillating patterns near the origin $\left|\beta^{(\mathrm{DI})}\right|=0$. 
Summary and discussion.-We have developed a theory and realized an experiment to characterize quantum light by means of phase space that functions for any type of detector and without performing a prior detector characterization. Our framework is based on the generating function derived from the properties of a balanced linear optical network, enabling our DAPS distribution, to be directly estimated from measured correlations and that applies to arbitrary states.

To demonstrate this concept, we showed that a single multiplexing step is already sufficient for applying our method. This renders it possible to verify the nonclassicality of multiphoton states based on DAPS distributions, which results in greater statistical significance than obtained with earlier approaches $[60,61]$, which themselves already outperformed previous quantifiers of nonclassicality. Moreover, our approach encompasses prominent phasespace quasiprobabilities and straightforwardly generalizes to multimode light.

Our general theory also includes more recent phasespace functions based on on-off detectors [82], constituting the special case $K=1$ and being applicable to off-the-shelf detectors (e.g., avalanche photodiodes in Geiger mode and single-photon nanowire detectors); to prove this, see the corresponding experiment with $S=1,2,3$ multiplexing steps [83]. Furthermore, recent advancements in detector technology (see, e.g., Refs. [84-87]) offer new photon counters to which our detector-agnostic framework is also readily applicable.

With our approach, we are further able to predict defining phase-space features of any states by measuring vacuum as a reference. Thus, we can compare a target state with the actually reconstructed DAPS distribution, thus enabling us to estimate other quantum properties as well. As a practical example, a state discrimination task based on our DAPS distributions resulted in distinguishing one- and two-photon states with almost unit certainty, despite high losses in our setup. It is also worth noting that our DAPS distribution includes the full quantum information of the state that is accessible with the detectors used and does not require demanding reconstruction algorithms and detection models.

Our experiment comprises state-of-the-art detectors combined with an advantageous method to create coherent states, well mode matched to our signal. As our method is detector agnostic, the detector efficiency need not be specified or even known; the number of data points merely has to be sufficient to produce statistically meaningful results. Also, our approach is not restricted to any specific states; currently, we are mainly limited by the available sources of nonclassical light.

In the future, recording the LO's phase would be beneficial for applying our scheme to phase-sensitive nonclassical states as well. Furthermore, generalizing other interferometric measurement schemes in a detector-agnostic manner is feasible, e.g., as done for on-off detectors [88]. In addition, we encounter the imperfections stemming from imbalances by assigning systematic errors. It may be possible to avoid this by using more sophisticated strategies [89].

Generalized phase-space distributions are becoming increasingly important in identifying vastly different notions of quantumness; see Refs. [49,50] for thorough overviews. To date, however, such universally applicable techniques are also highly dependent on the particular response of the detectors. Our DAPS approach, however, sets a precedence that such limitations can be overcome in theory and experiment.

In conclusion, our detector-agnostic framework provides a universally applicable approach to the robust characterization of quantum light in phase space under conditions where detailed knowledge of the measurement apparatus is not available, and forms a basis for future research.

The authors are grateful to William R. Clements for helpful discussions and Jelmer J. Renema for his assistance with the installation of the cryogenic infrastructure. The authors also thank Scott Glancy, Arik Avagyan, and Tim Bartley for valuable comments. The Integrated Quantum Optics group acknowledges financial support from the Gottfried Wilhelm Leibniz-Preis (Grant No. SI1115/3-1). This work received funding through the Networked Quantum Information Technologies (NQIT) hub (part of the UK National Quantum Technologies Programme) under Grant No. EP/N509711/1. G. S. T. acknowledges financial support from the Natural Sciences and Engineering Research Council of Canada and the Oxford Basil Reeve Graduate Scholarship. A. E. is supported by EPSRC (Grant No. EP/K034480/1 BLOQS). T. A. W. W. is supported by Fondation Wiener-Anspach. J. L. acknowledges the European Commission (H2020-FETPROACT2014 Grant No. QUCHIP). I. A. W. acknowledges ERC (Grant No. MOQUACINO). This work was supported by the Quantum Information Science Initiative (QISI).

*jan.sperling@uni-paderborn.de

[1] M. Krenn, M. Malik, T. Scheidl, R. Ursin, and A. Zeilinger, Quantum communication with photons, in Optics in Our Time (Springer, Cham, 2016), pp. 455-482.

[2] J. L. O'Brien, A. Furusawa, and J. Vučković, Photonic quantum technologies, Nat. Photonics 3, 687 (2009).

[3] T. C. Ralph and P. K. Lam, A bright future for quantum communications, Nat. Photonics 3, 671 (2009).

[4] D. F. Walls, Squeezed states of light, Nature (London) 306, 141 (1983).

[5] D. T. Smithey, M. Beck, M. G. Raymer, and A. Faridani, Measurement of the Wigner Distribution and the Density Matrix of a Light Mode Using Optical Homodyne Tomography: Application to Squeezed States and the Vacuum, Phys. Rev. Lett. 70, 1244 (1993). 
[6] G. Breitenbach, S. Schiller, and J. Mlynek, Measurement of the quantum states of squeezed light, Nature (London) 387, 471 (1997).

[7] A. I. Lvovsky, H. Hansen, T. Aichele, O. Benson, J. Mlynek, and S. Schiller, Quantum State Reconstruction of the SinglePhoton Fock State, Phys. Rev. Lett. 87, 050402 (2001).

[8] G. Harder, C. Silberhorn, J. Rehacek, Z. Hradil, L. Motka, B. Stoklasa, and L. L. Sánchez-Soto, Local Sampling of the Wigner Function at Telecom Wavelength with Loss-Tolerant Detection of Photon Statistics, Phys. Rev. Lett. 116, 133601 (2016).

[9] T. Kiesel, W. Vogel, V. Parigi, A. Zavatta, and M. Bellini, Experimental determination of a nonclassical GlauberSudarshan P function, Phys. Rev. A 78, 021804(R) (2008).

[10] K. Laiho, K. N. Cassemiro, D. Gross, and C. Silberhorn, Probing the Negative Wigner Function of a Pulsed Single Photon Point by Point, Phys. Rev. Lett. 105, 253603 (2010).

[11] T. Douce, A. Eckstein, S. P. Walborn, A. Z. Khoury, S. Ducci, A. Keller, T. Coudreau, and P. Milman, Direct measurement of the biphoton Wigner function through two-photon interference, Sci. Rep. 3, 3530 (2013).

[12] C. Baune, J. Fiurášek, and R. Schnabel, Negative Wigner function at telecommunication wavelength from homodyne detection, Phys. Rev. A 95, 061802(R) (2017).

[13] D.-G. Welsch, W. Vogel, and T. Opatrný, Homodyne detection and quantum-state reconstruction, Prog. Opt. 39, 63 (1999).

[14] C. Silberhorn, Detecting quantum light, Contemp. Phys. 48, 143 (2007).

[15] A. I. Lvovsky and M. G. Raymer, Continuous-variable optical quantum-state tomography, Rev. Mod. Phys. 81, 299 (2009).

[16] S. M. Tan, An inverse problem approach to optical homodyne tomography, J. Mod. Opt. 44, 2233 (1997).

[17] V. N. Starkov, A. A. Semenov, and H. V. Gomonay, Numerical reconstruction of photon-number statistics from photocounting statistics: Regularization of an ill-posed problem, Phys. Rev. A 80, 013813 (2009).

[18] T. Richter, Pattern functions used in tomographic reconstruction of photon statistics revisited, Phys. Lett. A 211, 327 (1996).

[19] U. Leonhard, M. Munroe, T. Kiss, T. Richter, and M. G. Raymer, Sampling of photon statistics and density matrix using homodyne detection, Opt. Commun. 127, 144 (1996).

[20] Z. Hradil, Quantum-state estimation, Phys. Rev. A 55, R1561(R) (1997).

[21] A. I. Lvovsky, Iterative maximum-likelihood reconstruction in quantum homodyne tomography, J. Opt. B 6, S556 (2004).

[22] R. Kosut, I. A. Walmsley, and H. Rabitz, Optimal experiment design for quantum state and process tomography and Hamiltonian parameter estimation, arXiv:quant-ph/ 0411093.

[23] J. Řeháček, D. Mogilevtsev, and Z. Hradil, Operational Tomography: Fitting of Data Patterns, Phys. Rev. Lett. 105, 010402 (2010).

[24] D. Mogilevtsev, A. Ignatenko, A. Maloshtan, B. Stoklasa, J. Rehacek, and Z. Hradil, Data pattern tomography: Reconstruction with an unknown apparatus, New J. Phys. 15, 025038 (2013).
[25] P. L. Kelley and W. H. Kleiner, Theory of electromagnetic field measurement and photoelectron counting, Phys. Rev. 136, A316 (1964).

[26] M. Fleischhauer and D. G. Welsch, Nonperturbative approach to multimode photodetection, Phys. Rev. A 44, 747 (1991).

[27] A. K. Jaiswal and G. S. Agarwal, Photoelectric detection with two-photon absorption, J. Opt. Soc. Am. 59, 1446 (1969).

[28] M. K. Akhlaghi, A. H. Majedi, and J. S. Lundeen, Nonlinearity in single photon detection: Modeling and quantum tomography, Opt. Express 19, 21305 (2011).

[29] S. V. Polyakov and A. L. Migdall, High accuracy verification of a correlated-photon-based method for determining photon-counting detection efficiency, Opt. Express 15, 1390 (2007).

[30] A. P. Worsley, H. B. Coldenstrodt-Ronge, J. S. Lundeen, P. J. Mosley, B. J. Smith, G. Puentes, N. Thomas-Peter, and I. A. Walmsley, Absolute efficiency estimation of photonnumber-resolving detectors using twin beams, Opt. Express 17, 4397 (2009).

[31] D. N. Klyshko, Use of two-photon light for absolute calibration of photoelectric detectors, Sov. J. Quantum Electron. 10, 1112 (1980).

[32] A. Luis and L. L. Sánchez-Soto, Complete Characterization of Arbitrary Quantum Measurement Processes, Phys. Rev. Lett. 83, 3573 (1999).

[33] J. Fiurášek, Maximum-likelihood estimation of quantum measurement, Phys. Rev. A 64, 024102 (2001).

[34] G. M. D'Ariano, L. Maccone, and P. Lo Presti, Quantum Calibration of Measurement Instrumentation, Phys. Rev. Lett. 93, 250407 (2004).

[35] A. Feito, J. S. Lundeen, H. Coldenstrodt-Ronge, J. Eisert, M. B. Plenio, and I. A. Walmsley, Measuring measurement: Theory and practice, New J. Phys. 11, 093038 (2009).

[36] H. B. Coldenstrodt-Ronge, J. S. Lundeen, K. L. Pregnell, A. Feito, B. J. Smith, W. Mauerer, C. Silberhorn, J. Eisert, M. B. Plenio, and I. A. Walmsley, A proposed testbed for detector tomography, J. Mod. Opt. 56, 432 (2009).

[37] J. J. Renema, G. Frucci, Z. Zhou, F. Mattioli, A. Gaggero, R. Leoni, M. J. A. de Dood, A. Fiore, and M. P. van Exter, Modified detector tomography technique applied to a superconducting multiphoton nanodetector, Opt. Express 20, 2806 (2012).

[38] J. Peřina, Jr., O. Haderka, V. Michálek, and M. Hamar, Absolute detector calibration using twin beams, Opt. Lett. 37, 2475 (2012).

[39] M. Bohmann, R. Kruse, J. Sperling, C. Silberhorn, and W. Vogel, Direct calibration of click-counting detectors, Phys. Rev. A 95, 033806 (2017).

[40] K. E. Cahill and R. J. Glauber, Density operators and quasiprobability distributions, Phys. Rev. 177, 1882 (1969).

[41] G.S. Agarwal and E. Wolf, Calculus for functions of noncommuting operators and general phase-space methods in quantum mechanics. II. Quantum mechanics in phase space, Phys. Rev. D 2, 2187 (1970).

[42] J. R. Klauder, Improved Version of Optical Equivalence Theorem, Phys. Rev. Lett. 16, 534 (1966).

[43] T. Kiesel and W. Vogel, Nonclassicality filters and quasiprobabilities, Phys. Rev. A 82, 032107 (2010). 
[44] K. Husimi, Some formal properties of the density matrix, Proc. Phys. Math. Soc. Jpn. 22, 264 (1940).

[45] O. Landon-Cardinal, L. C. G. Govia, and A. A. Clerk, Quantitative Tomography for Continuous Variable Quantum Systems, Phys. Rev. Lett. 120, 090501 (2018).

[46] D. F. Mundarain and J. Stephany, Husimi's $Q(\alpha)$ function and quantum interference in phase space, J. Phys. A 37, 3869 (2004).

[47] G. S. Agarwal, Nonclassical characteristics of the marginals for the radiation field, Opt. Commun. 95, 109 (1993).

[48] J. Park, Y. Lu, J. Lee, Y. Shen, K. Zhang, S. Zhang, M. S. Zubairy, K. Kim, and H. Nha, Revealing nonclassicality beyond Gaussian states via a single marginal distribution, Proc. Natl. Acad. Sci. U.S.A. 114, 891 (2017).

[49] J. Sperling and I. A. Walmsley, Quasiprobability representation of quantum coherence, Phys. Rev. A 97, 062327 (2018).

[50] J. Sperling and W. Vogel, Quasiprobability distributions for quantum-optical coherence and beyond, arXiv:1907.12427.

[51] G. S. Agarwal, Relation between atomic coherent-state representation, state multipoles, and generalized phasespace distributions, Phys. Rev. A 24, 2889 (1981).

[52] J. P. Dowling, G. S. Agarwal, and W. P. Schleich, Wigner distribution of a general angular-momentum state: Applications to a collection of two-level atoms, Phys. Rev. A 49, 4101 (1994).

[53] R. McConnell, H. Zhang, J. Hu, S. Čuk, and V. Vuletić, Entanglement with negative Wigner function of almost 3,000 atoms heralded by one photon, Nature (London) 519, 439 (2015).

[54] D. Leibfried, D. M. Meekhof, B. E. King, C. Monroe, W. M. Itano, and D. J. Wineland, Experimental Determination of the Motional Quantum State of a Trapped Atom, Phys. Rev. Lett. 77, 4281 (1996).

[55] A. Sanpera, R. Tarrach, and G. Vidal, Local description of quantum inseparability, Phys. Rev. A 58, 826 (1998).

[56] J. Sperling and W. Vogel, Representation of entanglement by negative quasiprobabilities, Phys. Rev. A 79, 042337 (2009).

[57] J. Sperling, E. Meyer-Scott, S. Barkhofen, B. Brecht, and C. Silberhorn, Experimental Reconstruction of Entanglement Quasiprobabilities, Phys. Rev. Lett. 122, 053602 (2019).

[58] S. Wallentowitz and W. Vogel, Unbalanced homodyning for quantum state measurements, Phys. Rev. A 53, 4528 (1996).

[59] H. Paul, P. Törmä, T. Kiss, and I. Jex, Photon Chopping: New Way to Measure the Quantum State of Light, Phys. Rev. Lett. 76, 2464 (1996).

[60] J. Sperling, W. R. Clements, A. Eckstein, M. Moore, J. J. Renema, W. S. Kolthammer, S. W. Nam, A. Lita, T. Gerrits, W. Vogel, G. S. Agarwal, and I. A. Walmsley, DetectorIndependent Verification of Quantum Light, Phys. Rev. Lett. 118, 163602 (2017).

[61] J. Sperling, A. Eckstein, W. R. Clements, M. Moore, J. J. Renema, W. S. Kolthammer, S. W. Nam, A. Lita, T. Gerrits, I. A. Walmsley, G. S. Agarwal, and W. Vogel, Identification of nonclassical properties of light with multiplexing layouts, Phys. Rev. A 96, 013804 (2017).

[62] See Supplemental Material at http://link.aps.org/ supplemental/10.1103/PhysRevLett.124.013605 for technical details about the experiment and theory, the data and error analysis, and additional results, which includes the Refs. [58,60,61,63-65].

[63] W. Vogel and D.-G. Welsch, Quantum Optics (Wiley-VCH, Weinheim, 2006).

[64] E. Wolf and C. L. Mehta, Determination of the Statistical Properties of Light from Photoelectric Measurements, Phys. Rev. Lett. 13, 705 (1964).

[65] J. Sperling, W. Vogel, and G. S. Agarwal, Quantum state engineering by click counting, Phys. Rev. A 89, 043829 (2014).

[66] R. J. Glauber, Coherent and incoherent states of the radiation field, Phys. Rev. 131, 2766 (1963).

[67] E.C. G. Sudarshan, Equivalence of Semiclassical and Quantum Mechanical Descriptions of Statistical Light Beams, Phys. Rev. Lett. 10, 277 (1963).

[68] U. M. Titulaer and R. J. Glauber, Correlation functions for coherent fields, Phys. Rev. 140, B676 (1965).

[69] L. Mandel, Non-classical states of the electromagnetic field, Phys. Scr. T12, 34 (1986).

[70] G. S. Agarwal, Quantum Optics (Cambridge University Press, Cambridge, England, 2012).

[71] Note that in our case, the $z$ parameter is not limited to values that correspond to $-1 \leq s \leq 1$ because of $\mathcal{K}$ being a finite set and, thus, guaranteeing convergence of $G_{z}$ for any $z$.

[72] A. Eckstein, A. Christ, P. J. Mosley, and C. Silberhorn, Highly Efficient Single-Pass Source of Pulsed SingleMode Twin Beams of Light, Phys. Rev. Lett. 106, 013603 (2011).

[73] M. Liscidini and J. E. Sipe, Stimulated Emission Tomography, Phys. Rev. Lett. 111, 193602 (2013).

[74] A. E. Lita, A. J. Miller, and S. W. Nam, Counting near infrared single-photons with $95 \%$ efficiency, Opt. Express 16, 3032 (2008).

[75] R. P. Welty and J. M. Martinis, A series array of DC SQUIDs, IEEE Trans. Magn. 27, 2924 (1991).

[76] E. Figueroa-Feliciano, B. Cabrera, A. J. Miller, S. F. Powell, T. Saab, and A. B. C. Walker, Optimal filter analysis of energy-dependent pulse shapes and its application to TES detectors, Nucl. Instrum. Methods Phys. Res., Sect. A 444, 453 (2000).

[77] P. C. Humphreys, B. J. Metcalf, T. Gerrits, T. Hiemstra, A. E. Lita, J. Nunn, S. W. Nam, A. Datta, W. S. Kolthammer, and I. A. Walmsley, Tomography of photon-number resolving continuous-output detectors, New J. Phys. 17, 103044 (2015).

[78] L. Mandel, subPoissonian photon statistics in resonance fluorescence, Opt. Lett. 4, 205 (1979).

[79] R. Short and L. Mandel, Observation of subPoissonian Photon Statistics, Phys. Rev. Lett. 51, 384 (1983).

[80] J. Sperling, W. Vogel, and G. S. Agarwal, subBinomial Light, Phys. Rev. Lett. 109, 093601 (2012).

[81] T. J. Bartley, G. Donati, X.-M. Jin, A. Datta, M. Barbieri, and I. A. Walmsley, Direct Observation of subBinomial Light, Phys. Rev. Lett. 110, 173602 (2013).

[82] A. Luis, J. Sperling, and W. Vogel, Nonclassicality PhaseSpace Functions: More Insight with Fewer Detectors, Phys. Rev. Lett. 114, 103602 (2015).

[83] M. Bohmann, J. Tiedau, T. Bartley, J. Sperling, C. Silberhorn, and W. Vogel, Incomplete Detection of Nonclassical PhaseSpace Distributions, Phys. Rev. Lett. 120, 063607 (2018). 
[84] C. Cahall, K. L. Nicolich, N. T. Islam, G. P. Lafyatis, A. J. Miller, D. J. Gauthier, and J. Kim, multiphoton detection using a conventional superconducting nanowire singlephoton detector, Optica 4, 1534 (2017).

[85] W. Guo, X. Liu, Y. Wang, Q. Wei, L. F. Wei, J. Hubmayr, J. Fowler, J. Ullom, L. Vale, M. R. Vissers, and J. Gao, Counting near infrared photons with microwave kinetic inductance detectors, Appl. Phys. Lett. 110, 212601 (2017).

[86] K. L. Nicolich, C. Cahall, N. T. Islam, G. P. Lafyatis, J. Kim, A. J. Miller, and D. J. Gauthier, Universal model for the turn-on dynamics of superconducting Nanowire singlephoton detectors, Phys. Rev. Applied 12, 034020 (2019).

[87] D. Zhu, M. Colangelo, C. Chen, B. A. Korzh, F. N. C. Wong, M. D. Shaw, and K. K. Berggren, Resolving photon numbers using a superconducting tapered nanowire detector, arXiv:1911.09485.

[88] J. Sperling, W. Vogel, and G. S. Agarwal, Balanced homodyne detection with on-off detector systems: Observable nonclassicality criteria, Europhys. Lett. 109, 34001 (2015).

[89] C. Lee, S. Ferrari, W. H. P. Pernice, and C. Rockstuhl, subPoisson-binomial light, Phys. Rev. A 94, 053844 (2016). 